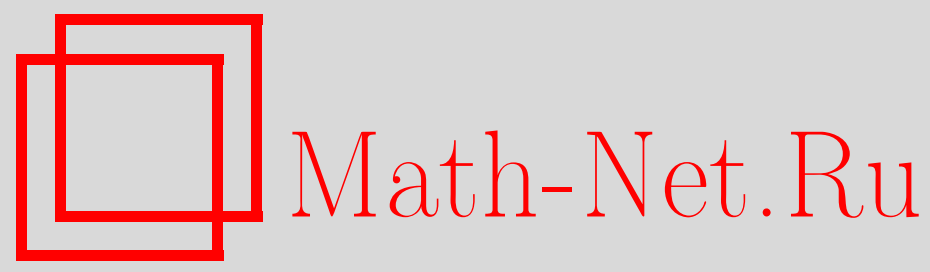

В. В. Арестов, В. Ю. Раевская, Одна экстремальная задача для алгебраических многочленов с нулевым средним значением на отрезке, Матем. заметки, 1997, том 62, выпуск $3,332-342$

DOI: https://doi.org/10.4213/mzm1615

Использование Общероссийского математического портала Math-Net.Ru подразумевает, что вы прочитали и согласны с пользовательским соглашением http://www.mathnet.ru/rus/agreement

Параметры загрузки:

IP : 52.6 .47 .48

26 апреля 2023 г., 04:50:04

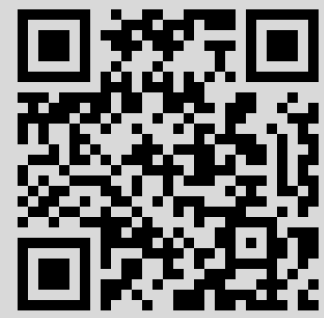




\title{
ОДНА ЭКСТРЕМАЛЬНАЯ ЗАДАЧА ДЛЯ АЛГЕБРАИЧЕСКИХ МНОГОЧЛЕНОВ С НУЛЕВЫМ СРЕДНИМ ЗНАЧЕНИЕМ НА ОТРЕЗКЕ
}

\author{
В.В. Арестов, В. Ю. Раевская
}

Пусть $\mathscr{P}_{n}^{0}(h)$ есть множество алгебраических многочленов порядка $n$ с действительными коэффициентами с нулевым средним значением (с весом $h)$ на отрезке $[-1,1]$ : $\int_{-1}^{1} h(x) p_{n}(x) d x=0$; здесь $h-$ функция, суммируемая, неотрицательная, отличная от нуля на множестве положительной меры на $[-1,1]$. Изучается задача о наименьшем возможном значении $i_{n}(h)=\inf \left\{\mu\left(p_{n}\right): p_{n} \in \mathscr{P}_{n}^{0}\right\}$ меры $\mu\left(p_{n}\right)=\operatorname{mes}\{x \in[-1,1]:$ $\left.p_{n}(x) \geqslant 0\right\}$ множества точек отрезка, в которых многочлен $p_{n} \in \mathscr{P}_{n}^{0}$ является неотрицательным. В работе найдено точное значение величины $i_{n}(h)$ при определенных ограничениях на вес $h$. Этим ограничениям удовлетворяет, в частности, вес Якоби $h^{(\alpha, \beta)}(x)=(1-x)^{\alpha}(1+x)^{\beta}$ при условии $-1<\alpha, \beta \leqslant 0$.

Библиография: 9 названий.

0. Введение. Пусть $\mathscr{P}_{n}$ есть множество алгебраических многочленов порядка $n$ с действительньми коэффициентами, а $\mathscr{P}_{n}^{0}=\mathscr{P}_{n}^{0}(h)-$ множество многочленов из $\mathscr{P}_{n} \mathrm{c}$ нулевым средним веса $h$ значением на отрезке $I=[-1,1]$ :

$$
\mathscr{P}_{n}^{0}(h)=\left\{p_{n} \in \mathscr{P}_{n}: \int_{-1}^{1} h(x) p_{n}(x) d x=0\right\}
$$

здесь $h$ - суммируемая и неотрицательная на $I$ функция, отличная от 0 на множестве положительной меры из $I$. Для многочлена $p_{n}$ введем множество $E\left(p_{n}\right)=\{x \in I$ : $\left.p_{n}(x) \geqslant 0\right\}$ точек отрезка $I=[-1,1]$, в которых многочлен $p_{n}$ неотрицательньй, и рассмотрим меру Лебега

$$
\mu\left(p_{n}\right)=\operatorname{mes} E\left(p_{n}\right)=\operatorname{mes}\left\{x \in I: p_{n}(x) \geqslant 0\right\}
$$

этого множества. При каждом натуральном $n$ определим величину

$$
i_{n}=i_{n}(h)=\inf \left\{\mu\left(p_{n}\right): p_{n} \in \mathscr{P}_{n}^{0}\right\}
$$

Работа выполнена при финансовой поддержке Международного научного фонда и Правительства России, грант J1T100. 
В данной работе будет найдено точное значение величины (2) при определенных ограничениях на вес $h$. Для веса $h \equiv 1$ задачу (2) изучал А. Г. Бабенко и показал [1, теорема 1.2], что

$$
\frac{1}{\sqrt{2} n^{2}} \leqslant i_{n}(1) \leqslant \frac{12}{n^{2}} .
$$

Оценку сверху он получил с помощью многочлена

$$
R_{2 k-1}(x)=\frac{P_{k}^{2}(x)}{x-x_{k}}
$$

где $P_{k}$ - многочлен Лежандра порядка $k$, а $x_{k}$ - его наибольший нуль. Как сказано в работе А.Г. Бабенко [1, с. 28], конструкция многочлена (3) заимствована им из работы С. Н. Бернштейна [2, с. 201], где, в частности, отмечено, что многочлен (3) имеет нулевое среднее значение на $[-1,1]$. Ниже мы покажем, что и в более общей ситуации экстремальный многочлен задачи (2) имеет аналогичньй вид.

Ранее Бабенко [3] вычислил аналог

$$
i_{n}^{*}=\min _{t_{n} \in \mathscr{T}_{n}^{0}} \operatorname{mes}\left\{x \in[0,2 \pi]: t_{n}(x) \geqslant 0\right\}
$$

величины $i_{n}(1)$ во множестве $\mathscr{T}_{n}^{0}$ тригонометрических полиномов $t_{n}$ порядка $n$ с нулевым средним значением на периоде:

$$
\int_{0}^{2 \pi} t_{n}(x) d x=0
$$

именно, он показал, что

$$
i_{n}^{*}=\frac{2 \pi}{n+1} .
$$

Последняя задача возникла в работе Л.В. Тайкова [4] при исследовании точной константы в неравенстве между нормами тригонометрического полинома в пространствах $C[0,2 \pi]$ и $L(0,2 \pi)$. Как видно из работы Бабенко [5], подобные задачи естественным образом появляются при доказательстве точных неравенств Джексона для наилучших среднеквадратических приближений периодических функций тригонометрическими полиномами. Задачи, близкие (2), (4), возникают также в других разделах математики и, в частности, при получении оценок плотности упаковок множеств (см. работы [6], [7] и приведенную там библиографию).

1. Вспомогательные утверждения. Нетрудно убедиться, что экстремальный многочлен задачи (2), на котором достигается нижняя грань, существует. Действительно, у многочленов

$$
p_{n}(x)=\sum_{k=0}^{n} a_{k} x^{k}
$$

из множества $\mathscr{P}_{n}^{0}$ коэффициенты $a_{1}, \ldots, a_{n}$ можно выбирать произвольно, после чего коэффициент $a_{0}$ однозначно определяется из условия ортогональности

$$
\int_{-1}^{1} h(x) p_{n}(x) d x=0 .
$$


Функция (1) однородна по коэффициентам многочлена $p_{n}$ (точнее, она не изменяется при умножении многочлена на положительную константу), поэтому в (2) можно ограничиться многочленами (5) из $\mathscr{P}_{n}^{0}$ с условием

$$
\sum_{k=1}^{n}\left|a_{k}\right|=1
$$

Таким образом, задача (2) является задачей минимизации непрерьвной функции коэффициентов $a_{1}, \ldots, a_{n}$ многочлена (5) на компактном множестве (6), и следовательно, нижняя грань в (2) достигается, т.е. в (2) существует экстремальньй многочлен.

ЛЕмма 1. Если для многочлена $p_{n} \in \mathscr{P}_{n}^{0}$ множсество

$$
e\left(p_{n}\right)=\left\{x \in I: p_{n}(x)>0\right\}
$$

несвязно, то $p_{n}$ не является әкстремальным, т.е. $\mu\left(p_{n}\right)>i_{n}$.

ДоКАЗАТЕЛЬСТво. Пусть $p_{n}$ есть многочлен из множества $\mathscr{P}_{n}^{0}$, для которого множество $e\left(p_{n}\right)=\left\{x \in I: p_{n}(x)>0\right\}$ несвязно. Докажем, что тогда $\mu\left(p_{n}\right)>i_{n}$. Множество $e\left(p_{n}\right)$ есть объединение двух или более непересекающихся промежутков. Отсюда следует, что $p_{n}$ можно представить в виде

$$
p_{n}(x)=\left(x-x_{1}\right)\left(x-x_{2}\right) p_{n-2}(x),
$$

где $x_{1} \leqslant x_{2}-$ нули многочлена $p_{n}$ из интервала $(-1,1)$, а $p_{n-2}-$ многочлен порядка $n-2$ такой, что существуют действительные числа $a, b$ со свойствами $-1 \leqslant a<x_{1} \leqslant x_{2}<$ $b \leqslant 1$ и $p_{n-2}(x) \geqslant 0$ при $a \leqslant x \leqslant b$ (при этом, возможно, что $x_{1}=x_{2}$ есть кратньй корень $\left.p_{n}\right)$.

Для вещественных чисел $\varepsilon, \delta$ определим многочлен

$$
p_{n}^{\varepsilon, \delta}(x)=\left(x-\left(x_{1}+\varepsilon\right)\right)\left(x-\left(x_{2}+\delta\right)\right) p_{n-2}(x) .
$$

Потребуем, чтобы он принадлежал множеству $\mathscr{P}_{n}^{0}(h)$; это налагает на $\varepsilon$ и $\delta$ условие

$\varepsilon \int_{-1}^{1} h(x)\left(x-x_{2}\right) p_{n-2}(x) d x+\delta \int_{-1}^{1} h(x)\left(x-x_{1}\right) p_{n-2}(x) d x-\varepsilon \delta \int_{-1}^{1} h(x) p_{n-2}(x) d x=0$.

Обозначим коэффициенты при $\varepsilon, \delta$ и - $\varepsilon \delta$ последнего соотношения через $A, B$ и $C$ соответственно; в результате оно примет вид

$$
\varepsilon A+\delta B+\varepsilon \delta C=0
$$

В зависимости от коэффициентов $A, B, C$ уравнение $(7)$ задает в плоскости $\varepsilon, \delta$ гиперболу $(A B C \neq 0)$, пару прямых $(C \neq 0, A B=0)$, одну прямую $\left(C=0, A^{2}+B^{2} \neq 0\right)$ или всю плоскость $\left(A^{2}+B^{2}+C^{2}=0\right)$. Ясно, что точка $(0,0)$ принадлежит кривой, заданной уравнением (7). Убедимся, что при определенных условиях на коэффициенты $A, B, C$ (т.е. на многочлен $\left.p_{n}\right)$ в любой окрестности точки $(0,0)$ в плоскости $\varepsilon, \delta$ найдется точка, принадлежашая кривой и удовлетворяющая условию $\delta>\varepsilon$. Очевидно, что в случае $A^{2}+B^{2}+C^{2}=0$ и в случае $C \neq 0, A B=0$ такая точка найдется. В случае $C=0, A^{2}+B^{2} \neq 0$ для существования такой точки необходимо и достаточно, чтобы 
прямая (7) не совпадала с прямой $\varepsilon=\delta$, т.е. чтобы $A+B \neq 0$. Условие $A+B \neq 0$ обеспечивает существование нужной точки и в случае $A B C \neq 0$, ибо если $A+B \neq 0$, то касательная к гиперболе $(7)$ в точке $(0,0)$ не совпадает с прямой $\varepsilon=\delta$. Таким образом, если многочлен $p_{n}$ таков, что $A+B \neq 0$, то найдется пара чисел $\varepsilon$ и $\delta$, удовлетворяющая условиям $\varepsilon<\delta,(7)$ и условию $a<x_{1}+\varepsilon<x_{2}+\delta<b$. Тогда имеем $p_{n}^{\varepsilon, \delta} \in \mathscr{P}_{n}^{0}(h)$, $\mu\left(p_{n}^{\varepsilon, \delta}\right)=\mu\left(p_{n}\right)+\varepsilon-\delta$, а следовательно, $\mu\left(p_{n}^{\varepsilon, \delta}\right)<\mu\left(p_{n}\right)$ и тем более $\mu\left(p_{n}\right)>i_{n}$.

Предположим теперь, что $A+B=0$. Это условие означает, что для многочлена $p_{n}$ (а точнее, для многочлена $p_{n-2}$ ) имеет место равенство

$$
\int_{-1}^{1} h(x)\left(x-x_{2}\right) p_{n-2}(x) d x+\int_{-1}^{1} h(x)\left(x-x_{1}\right) p_{n-2}(x) d x=0 .
$$

или, что то же самое, равенство

$$
\int_{-1}^{1} h(x)\left(x-\frac{x_{1}+x_{2}}{2}\right) p_{n-2}(x) d x=0 .
$$

Введем многочлен

$$
p_{n}^{c}(x)=p_{2}(x) p_{n-2}(x)
$$

где

$$
p_{2}(x)=p_{2}^{c}(x)=\left(x-x_{1}\right)\left(x-x_{2}\right)+c\left(x-\frac{x_{1}+x_{2}}{2}\right),
$$

а $c$ - вещественньй параметр. При любом значении параметра $c$ многочлен $p_{n}^{c}$ принадлежит множеству $\mathscr{P}_{n}^{0}(h)$. Дискриминант $D$ многочлена $p_{2}^{c}$ есть неотрицательное вещественное число, а именно, $4 D=c^{2}+\left(x_{2}-x_{1}\right)^{2}$. Следовательно, $p_{2}^{c}$ имеет два вещественных корня $x_{1}^{c}$ и $x_{2}^{c}$, расстояние между которьми равно $\sqrt{c^{2}+\left(x_{2}-x_{1}\right)^{2}}$. Эти корни непрерьвно зависят от параметра $c$ и при $c=0$ совпадают с точками $x_{1}, x_{2}$. Поэтому для достаточно малых (отличных от нуля) значений $c$ корни $x_{1}^{c}$ и $x_{2}^{c}$ будут лежать на интервале $(a, b)$. При таком предположении имеем

$$
\mu\left(p_{n}\right)-\mu\left(p_{n}^{c}\right)=\sqrt{c^{2}+\left(x_{2}-x_{1}\right)^{2}}-\left(x_{2}-x_{1}\right)>0,
$$

а значит, $\mu\left(p_{n}\right)>\mu\left(p_{n}^{c}\right)$. Лемма доказана полностью.

Согласно только что доказанной лемме в (2) можно ограничиться многочленами $p_{n} \in$ $\mathscr{P}_{n}^{0}(h)$, для которых множество $e\left(p_{n}\right)=\left\{x \in[-1,1]: p_{n}(x)>0\right\}$ есть (один) промежуток; обозначим совокупность многочленов с таким свойством через $\mathscr{P}_{n}^{*}=\mathscr{P}_{n}^{*}(h)$. Таким образом, справедливо утверждение.

ТЕОРемА 1. При любом натуральном $n$ для произвольного суммируемого неотрицательного веса $h$ имеет место равенство

$$
i_{n}(h)=\min \left\{\mu\left(p_{n}\right): p_{n} \in \mathscr{P}_{n}^{*}(h)\right\}
$$

Обозначим, далее, через $\mathscr{P}_{n}^{1}=\mathscr{P}_{n}^{1}(h)$ множество многочленов $p_{n} \in \mathscr{P}_{n}^{0}(h)$, имеющих ровно одну перемену знака на интервале $(-1,1)$. Для каждого натурального $n$ определим величину

$$
j_{n}=j_{n}(h)=\inf \left\{\mu\left(p_{n}\right): p_{n} \in \mathscr{P}_{n}^{1}(h)\right\}
$$


которая, как будет показано ниже, при определенных ограничениях на вес $h$ совпадает c (2). Значение величины (8) и соответствуюший экстремальньй многочлен зависят, в частности, от четности параметра $n$.

Пусть $U_{k}, V_{k}, W_{k}$ - многочлены $k$-й степени, $k \geqslant 1$, отличные от тождественного нуля (например, с единичным старшим коэффициентом), ортогональные на отрезке $[-1,1]$ всем многочленам степени меньшей $k$, соответственно с весами

$$
h(x), \quad h(x)(1+x), \quad h(x)(1-x),
$$

и пусть $-1<u_{1}<\cdots<u_{k}<1,-1<v_{1}<\cdots<v_{k}<1,-1<w_{1}<\cdots<w_{k}<1-$ нули этих многочленов.

ЛЕмма 2. Если число $п$ нечетное, $n=2 k-1, k \geqslant 1$, то

$$
j_{n}(h)=\min \left\{1-u_{k}, 1+u_{1}\right\}
$$

и в зависимости от того, какая из двух величин в (9) является наименьшей, әкстремальным в (8) будет соответственно один из многочленов

$$
\frac{U_{k}^{2}(x)}{x-u_{k}}, \quad \frac{U_{k}^{2}(x)}{u_{1}-x} .
$$

Если число п четное, $n=2 k, k \geqslant 1$, то

$$
j_{n}(h)=\min \left\{1-v_{k}, 1+w_{1}\right\}
$$

и в зависимости от того, какая из двух величин в (11) является наименьшей, әкстремальным в (8) будет соответственно один из многочленов

$$
\frac{(1+x) V_{k}^{2}(x)}{x-v_{k}}, \quad \frac{(1-x) W_{k}^{2}(x)}{w_{1}-x} .
$$

После того, как эта заметка была подготовлена к печати, вышли работы [6], [7]. Из результатов этих работ можно получить утверждения леммы 2. Однако, авторы сочли полезным привести независимое доказательство леммы.

ДокАЗАТЕЛЬСтво лЕммы 2. Вначале исследуем случай нечетного $n=2 k-1$, $k \geqslant 1$. Для многочленов $p_{n} \in \mathscr{P}_{n}$ справедлива квадратурная формула типа Гаусса (см., например, [8, гл. 7, с. 95-101])

$$
\int_{-1}^{1} h(x) p_{n}(x) d x=\sum_{m=1}^{k} A_{m} p_{n}\left(u_{m}\right)
$$

в которой $\left\{u_{m}\right\}_{m=1}^{k}$ - нули многочлена $U_{k}$, a $\left\{A_{m}\right\}_{m=1}^{k}$ - некоторые положительные коэффициенты (веса). В частности, для многочленов $p_{n} \in \mathscr{P}_{n}^{0}$ имеем

$$
\sum_{m=1}^{k} A_{m} p_{n}\left(u_{m}\right)=\int_{-1}^{1} h(x) p_{n}(x) d x=0
$$


Если многочлен $p_{n}$ не есть тождественньй нуль, то все узлы $u_{m}$ одновременно не могут лежать на интервале знакопостоянства $p_{n}$, ибо из (14) в силу положительности весов $A_{m}$ следовало бы, что каждая из точек $u_{m}$ является кратным нулем $p_{n}$, и значит, этот многочлен должен иметь порядок, не меньший $2 k$, хотя в данном случае $n=2 k-1$. Пусть к тому же многочлен $p_{n}$ принадлежит $\mathscr{P}_{n}^{1}$ и $x^{*}$ - точка перемены знака $p_{n}$. Тогда, если правее точки $x^{*}$ этот многочлен неотрицательный (а следовательно, левее точки $x^{*}$ он неположительный), то из только что проведенных рассуждений следует, что неможет выполняться неравенство $x^{*}>u_{k}$, и значит, $x^{*} \leqslant u_{k}$, апотому $\mu\left(p_{n}\right) \geqslant 1-u_{k}$. Если же многочлен $p_{n}$ является неотрицательньм левее точки $x^{*}$, то $x^{*} \geqslant u_{1}$ и $\mu\left(p_{n}\right) \geqslant 1+u_{1}$. Таким образом, для любого многочлена $p_{n} \in \mathscr{P}_{n}^{1}$ имеет место неравенство

$$
\mu\left(p_{n}\right) \geqslant \min \left\{1-u_{k}, 1+u_{1}\right\}
$$

Оба многочлена (10) имеют порядок $n=2 k-1$ и обнуляют правую, а значит, и левую части (13), т.е. принадлежат множеству $\mathscr{P}_{n}^{0}$. Очевидно, что на одном из этих многочленов неравенство (15) обращается в равенство (в соответствии с тем, какая из величин правой части (15) является наименњшей). Из приведенных рассуждений нетрудно получить, что указанные многочлены с точностью до положительного коэффициента являются единственными экстремальными. Тем самым, утверждение леммы при $n=2 k-1$ доказано.

Пусть теперь число $n$ четное, $n=2 k, k \geqslant 1$. Воспользуемся квадратурной формулой Гаусса с одним наперед заданным узлом $v_{0}=-1$ [8, гл. 9, с. 161-180]

$$
\int_{-1}^{1} h(x) p_{n}(x) d x=A_{0} p_{n}(-1)+\sum_{m=1}^{k} A_{m} p_{n}\left(v_{m}\right)
$$

и аналогичной формулой с фиксированньм узлом $w_{m+1}=1$

$$
\int_{-1}^{1} h(x) p_{n}(x) d x=B_{m+1} p_{n}(1)+\sum_{m=1}^{k} B_{m} p_{n}\left(w_{m}\right)
$$

являющимися точными на полиномах $p_{n}$ порядка $n$. В этих формулах $\left\{v_{m}\right\}_{m=1}^{k}-$ нули многочлена $V_{k},\left\{w_{m}\right\}_{m=1}^{k}$ - нули многочлена $W_{k}$, a $\left\{A_{m}\right\}_{m=0}^{k}$ и $\left\{B_{m}\right\}_{m=1}^{k+1}-$ некоторые положительные веса. Для многочленов $p_{n} \in \mathscr{P}_{n}^{0}$ как следствие будем иметь

$$
\begin{gathered}
A_{0} p_{n}(-1)+\sum_{m=1}^{k} A_{m} p_{n}\left(v_{m}\right)=0 \\
B_{m+1} p_{n}(1)+\sum_{m=1}^{k} B_{m} p_{n}\left(w_{m}\right)=0 .
\end{gathered}
$$

Пусть, в частности, многочлен $p_{n}$ принадлежит множеству $\mathscr{P}_{n}^{1}$ и $x^{*}$ - точка перемены знака $p_{n}$. Тогда если $p_{n}$ неотрицательный правее точки $x^{*}$, то, как и вьше, из соотношения (16) делаем вьвод, что $x^{*} \leqslant v_{k}$ и $\mu\left(p_{n}\right) \geqslant 1-v_{k}$. Если же $p_{n}$ неотрицательный левее точки $x^{*}$, то аналогичньм образом из соотношения (17) выводим, что $x^{*} \geqslant w_{1}$ и $\mu\left(p_{n}\right) \geqslant 1+w_{1}$. Таким образом, в случае четного $n$ для любого многочлена $p_{n} \in \mathscr{P}{ }_{n}^{1}$ имеет место неравенство

$$
\mu\left(p_{n}\right) \geqslant \min \left\{1-v_{k}, 1+w_{1}\right\} .
$$


Многочлены $(12)$ имеют порядок $n=2 k$ и обнуляют правые части формул $(13),(14)$ и, значит, принадлежат множеству $\mathscr{P}_{n}^{0}$. На одном из этих многочленов неравенство (18) обрашается в равенство (в соответствии с тем, какая из величин правой части (18) является наименьшей). Так же, как и для нечетного $n$, указанные многочлены с точностью до положительного коэффициента являются единственньпи экстремальными. Тем самым, утверждение леммы проверено и для $n$ четного. Лемма доказана полностью.

2. Основной результат. В этом пункте будет дано решение задачи (2) при дополнительных ограничениях на вес $h$. Весу $h$, т.е. неотрицательной, измеримой, суммируемой на отрезке $[-1,1]$ функции $h$, сопоставим функции

$$
h^{-}(t)=h(t-1), \quad h^{+}(t)=h(1-t),
$$

определенные при $t \in[0,2]$.

ОПРЕДЕЛЕНИЕ. Будем говорить, что вес $h$ обладает $\Theta$-свойством, если функция $h$ положительная, непрерьвная на интервале $(-1,1)$ и при любом $\theta \in(0,1)$ функции

$$
\frac{h^{-}(t)}{h^{-}(\theta t)}, \frac{h^{+}(t)}{h^{+}(\theta t)}
$$

не убывают по переменному $t$ на интервале $(0,2)$.

ТЕорема 2. Если вес $h$ обладает $\Theta$-свойством, то при любом натуральном $n$ имеет место равенство

$$
i_{n}(h)=j_{n}(h) .
$$

Напомним, что величина $j_{n}(h)$ была вычислена в лемме 2, где, в частности, указаны экстремальные многочлены задачи (8), а следовательно (в условиях теоремы), и задачи (2).

ДокАЗАТЕЛЬСТво. Поскольку $\mathscr{P}_{n}^{1}(h) \subset \mathscr{P}_{n}^{0}(h)$, то $i_{n}(h) \leqslant j_{n}(h)$. Следовательно, для доказательства теоремы нужно проверить обратное неравенство. В силу теоремы 1 и определения (8) величины $j_{n}$ достаточно убедиться, что для любого многочлена $p_{n} \in \mathscr{P}_{n}^{*}(h) \backslash \mathscr{P}_{n}^{1}(h)$ имеет место неравенство $\mu\left(p_{n}\right) \geqslant j_{n}(h)$. Пусть $p_{n}$ именно такой многочлен и $x_{1}^{*}<x_{2}^{*}$ - точки перемены знака этого многочлена на $(-1,1)$; при этом имеем $p_{n}(x)>0$, если $x \in\left(x_{1}^{*}, x_{2}^{*}\right)$, и $p_{n}(x) \leqslant 0$, если $-1 \leqslant x \leqslant x_{1}^{*}$ или $x_{2}^{*} \leqslant x \leqslant 1$, и как следствие $\mu\left(p_{n}\right)=x_{2}^{*}-x_{1}^{*}$. Многочлен $p_{n}$ удовлетворяет условию

$$
\int_{-1}^{1} h(x) p_{n}(x) d x=0
$$

поэтому существует точка $c \in\left[x_{1}^{*}, x_{2}^{*}\right]$ такая, что

$$
\int_{-1}^{c} h(x) p_{n}(x) d x=0, \quad \int_{c}^{1} h(x) p_{n}(x) d x=0 .
$$

Введем многочлен

$$
p_{n}^{c}(x)=p_{n}\left(x \frac{c+1}{2}+\frac{c-1}{2}\right)
$$


Этот многочлен лишш в одной точке

$$
x^{c}=\frac{2}{c+1}\left(x_{1}^{*}-\frac{c-1}{2}\right)
$$

интервала $(-1,1)$ меняет знак, причем с минуса на плюс, и следовательно,

$$
\mu\left(p_{n}^{c}\right)=1-x^{c}=\frac{2}{c+1}\left(c-x_{1}^{*}\right)
$$

Определим, далее, на отрезке $[-1,1]$ вес

$$
h^{c}(x)=h\left(x \frac{c+1}{2}+\frac{c-1}{2}\right) .
$$

В силу выбора (20) точки $с$ имеем

$$
\int_{-1}^{1} h^{c}(x) p_{n}^{c}(x) d x=\frac{2}{c+1} \int_{-1}^{c} h(x) p_{n}(x) d x=0
$$

и, таким образом, $p_{n}^{c} \in \mathscr{P}_{n}^{1}\left(h^{c}\right)$. Нас интересует знак интеграла

$$
Z=\int_{-1}^{1} h(x) p_{n}^{c}(x) d x
$$

Запишем этот интеграл в виде

$$
Z=\int_{-1}^{1} s(x) h^{c}(x) p_{n}^{c}(x) d x
$$

где

$$
s(x)=\frac{h(x)}{h^{c}(x)} .
$$

В силу $\Theta$-свойства веса $h$ функция $s$ неотрицательна и не убывает на $(-1,1)$; можно считать, что она обладает этим свойством на промежутке $[-1,1)$. Нетрудно видеть, что функция

$$
H(x)=\int_{-1}^{x} h^{c}(t) p_{n}^{c}(t) d t
$$

удовлетворяет условиям

$$
H(-1)=H(1)=0
$$

не возрастает на отрезке $\left[-1, x^{c}\right]$ и не убывает на $\left[x^{c}, 1\right]$, а значит,

$$
H(x) \leqslant 0, \quad x \in[-1,1]
$$

Убедимся, что перечисленные свойства функций $s$ и $H$ обеспечивают неравенство

$$
Z \geqslant 0
$$


Проверим этот факт вначале в предположении, что функция $s$ ограничена (сверху) на $[-1,1)$. Доопределим ее в точке $x=1$ так, чтобы она осталась монотонной на отрезке $[-1,1]$ и возьмем интеграл $(22)$ по частям; в результате получим представление

$$
Z=Z_{1}-Z_{2}
$$

где

$$
Z_{1}=\left.s(x) H(x)\right|_{-1} ^{1}, \quad Z_{2}=\int_{-1}^{1} H(x) d s(x) .
$$

Свойство (23) функции $H$ влечет $Z_{1}=0$. Далее, в силу монотонности функции $s$ и свойства (24) функции $H$ заключаем, что $Z_{2} \leqslant 0$, а значит, имеет место неравенство (25). Допустим теперь, что функция $s$ неограничена сверху на $[-1,1)$. При $N>0$ введем функцию $s_{N}=\min (s, N)$, являющуюся срезкой $s$. К интегралу

$$
Z(N)=\int_{-1}^{1} s_{N}(x) h_{c}(x) p_{n}^{c}(x) d x
$$

применимо только что доказанное утверждение, согласно которому $Z(N) \geqslant 0$. Кроме того, $Z(N) \rightarrow Z$ при $N \rightarrow \infty$, а значит, и в этом случае имеет место (25).

В силу свойства (25) существует константа $C \geqslant 0$ такая, что

$$
\int_{-1}^{1} h(x)\left(p_{n}^{c}(x)-C\right) d x=0
$$

Многочлен $q_{n}=p_{n}^{c}(x)-C$, очевидно, принадлежит множеству $\mathscr{P}_{n}^{1}(h)$, и потому $\mu\left(q_{n}\right) \geqslant$ $j_{n}(h)$. Кроме того, поскольку $C \geqslant 0$, то

$$
\mu\left(q_{n}\right) \leqslant 1-x_{1}^{c}=\mu\left(p_{n}^{c}\right)
$$

значит,

$$
\mu\left(p_{n}^{c}\right) \geqslant j_{n}(h)
$$

что в силу (21) дает оценку

$$
\operatorname{mes}\left\{x \in[-1, c]: p_{n}(x) \geqslant 0\right\}=c-x_{1}^{*} \geqslant \frac{c+1}{2} j_{n}(h)
$$

$\operatorname{Bec} \bar{h}(x)=h(-x)$ и многочлен $\bar{p}_{n}(x)=p_{n}(-x)$ обладают на $[-1,1]$ всеми свойствами пары $h, p_{n}$. Нетрудно понять, что аналог соотношения $(26)$ для веса $\bar{h}$ и многочлена $\bar{p}_{n}$ будет иметь вид

$$
\operatorname{mes}\left\{x \in[c, 1]: p_{n}(x) \geqslant 0\right\}=x_{2}^{*}-c \geqslant \frac{1-c}{2} j_{n}(h)
$$

Складьвая неравенства $(26)$ и $(27)$, получаем нужную оценку $\mu\left(p_{n}\right) \geqslant j_{n}(h)$. Теорема доказана. 
3. Обсуждение основной задачи для веса Якоби. Исходная задача (2) представляет интерес, в частности, для веса Якоби

$$
h(x)=h^{(\alpha, \beta)}(x)=(1-x)^{\alpha}(1+x)^{\beta}
$$

при естественных ограничениях $\alpha>-1, \beta>-1$, обеспечивающих суммируемость этого веса. Ортогональную на отрезке $[-1,1]$ с весом $h^{(\alpha, \beta)}$ систему алгебраических многочленов образуют классические многочлены Якоби $P_{k}^{(\alpha, \beta)}, k \geqslant 0$ [9]. Обозначим через $x_{1}(\alpha, \beta)$ и $x_{k}(\alpha, \beta)$ соответственно наименьший и наибольший нули многочлена $P_{k}^{(\alpha, \beta)}$; они связаны соотношением $x_{1}(\alpha, \beta)=-x_{k}(\beta, \alpha)$.

Нетрудно понять, что величины

$$
i_{n}(\alpha, \beta)=i_{n}\left(h^{(\alpha, \beta)}\right), \quad j_{n}(\alpha, \beta)=j_{n}\left(h^{(\alpha, \beta)}\right)
$$

не изменяются при перестановке параметров $\alpha$ и $\beta$, поэтому можно считать, что $\alpha \leqslant \beta$. Утверждение леммы 2 принимает в данном частном случае следующий вид.

Лемма 3. Пусть параметры $\alpha, \beta$ удовлетворяют условиям $-1<\alpha \leqslant \beta$. Тогда если число п нечетное, $n=2 k-1, k \geqslant 1$, то $j_{n}(\alpha, \beta)=1-x_{k}(\alpha, \beta)$ и многочлен

$$
\frac{\left(P_{k}^{(\alpha, \beta)}(x)\right)^{2}}{x-x_{k}(\alpha, \beta)}
$$

является экстремальным. Если число $n$ четное, $n=2 k, k \geqslant 1$, mо $j_{n}(\alpha, \beta)=$ $1-x_{k}(\alpha, \beta+1)$ имногочлен

$$
\frac{(1+x)\left(P_{k}^{(\alpha, \beta+1)}(x)\right)^{2}}{x-x_{k}(\alpha, \beta+1)} .
$$

является әкстремальным.

ДокАЗАТЕльство. Известно [9, теорема 6.21 .1$]$, что нули многочлена Якоби $P_{k}^{(\alpha, \beta)}$ и, в частности, наибольший $x_{k}(\alpha, \beta)$ и наименьший $x_{1}(\alpha, \beta)$ нули по переменному $\alpha$ убывают, а по переменному $\beta$ возрастают. Из этого легко сделать вьвод, что (в предположении $\alpha \leqslant \beta$ ) имеет место неравенство

$$
1-x_{k}(\alpha, \beta) \leqslant 1+x_{1}(\alpha, \beta)=1-x_{k}(\beta, \alpha) .
$$

Поэтому в случае нечетного $n$ утверждение леммы 3 вытекает из соответствующего утверждения леммы 2 .

В силу леммы 2 для четного $n=2 k$ справедливо равенство

$$
j_{n}(\alpha, \beta)=\min \left\{1-x_{k}(\alpha, \beta+1), 1+x_{1}(\alpha+1, \beta)\right\}
$$

Для доказательства леммы в этом случае нужно убедиться, что если $-1<\alpha \leqslant \beta$, то

$$
1-x_{k}(\alpha, \beta+1) \leqslant 1+x_{1}(\alpha+1, \beta) .
$$

Введем обозначение $l_{k}(\alpha, \beta)=1-x_{k}(\alpha, \beta)$; эта функция по переменному $\alpha$ растет, а по переменному $\beta$ убьвает. Поэтому поскольку $-1<\alpha \leqslant \beta \leqslant 0$, то

$$
1+x_{1}(\alpha+1, \beta)=l_{k}(\beta, \alpha+1) \geqslant l_{k}(\alpha, \alpha+1) \geqslant l_{k}(\alpha, \beta+1) .
$$


Неравенство (31) проверено. Лемма доказана полностью.

Для веса (28) функции (19) принимают вид

$$
\frac{1}{\theta^{\beta}}\left(\frac{2-t}{2-\theta t}\right)^{\alpha}, \quad \frac{1}{\theta^{\alpha}}\left(\frac{2-t}{2-\theta t}\right)^{\beta}
$$

и являются неубьвающими по $t \in(0,2)$ при $\alpha \leqslant 0, \beta \leqslant 0$. Следовательно, если $-1<\alpha \leqslant 0,-1<\beta \leqslant 0$, то вес (28) обладает $\Theta$-свойством.

Как следствие теоремы 2 и леммы 3 для величины $i_{n}(\alpha, \beta)=i_{n}\left(h^{(\alpha, \beta)}\right)$ имеем следующее утверждение.

ТЕОрема 3. Пусть параметры $\alpha, \beta$ удовлетворяют условиям $-1<\alpha \leqslant \beta \leqslant 0$. Тогда если число $n$ нечетное, $n=2 k-1, k \geqslant 1$, то

$$
i_{n}(\alpha, \beta)=1-x_{k}(\alpha, \beta)
$$

и многочлен (29) является әкстремальным. Если число $n$ четное, $n=2 k, k \geqslant 1$, mo

$$
i_{n}(\alpha, \beta)=1-x_{k}(\alpha, \beta+1)
$$

и многочлен (30) является әкстремальным.

При фиксированных $\alpha, \beta(-1<\alpha \leqslant \beta \leqslant 0)$ величина $i_{n}(\alpha, \beta)$ имеет следующее асимптотическое поведение по $n$ :

$$
i_{n}(\alpha, \beta)=\frac{2 \rho_{\alpha}^{2}}{n^{2}}+O\left(\frac{1}{n^{3}}\right), \quad n \rightarrow \infty,
$$

где $\rho_{\alpha}-$ первый положительный нуль функции Бесселя $J_{\alpha}$ порядка $\alpha$. Это утверждение есть следствие формул $(32),(33)$ и хорошо известной асимптотики поведения нулей полиномов Якоби [9, теорема 8.1.2].

\section{СПИСОК ЦИТИРОВАННОЙ ЛИТЕРАТУРЫ}

[1] Бабенко А. Г. Экстремальные свойства полиномов и точные оценки среднеквадратичных приближений. Дисс. ... к. ф.-м. н. Свердловск, 1987.

[2] Бернштейн С. Н. О формулах квадратур Котеса и Чебышева // Собрание сочинений (в 4-х томах). Т. 2. М., 1954. С. 200-204.

[3] Бабенко А. Г. Об одной экстремальной задаче для полиномов // Матем. заметки. 1984. T. 35. № 3. C. $349-356$.

[4] Тайков Л. В. Один круг экстремальных задач для тригонометрических полиномов // УМН. 1965. T. 20. № 3. C. 205-211.

[5] Бабенко А. Г. Неравенство Джексона для среднеквадратичных приближений периодических функций тригонометрическими полиномами на равномерной сетке // Матем. заметки. 1988. T. 43. № 4. C. 460-473.

[6] Fazekas G., Levinstein V.I. On upper bounds for code distance and covering radius of designs in polynomial metric spaces // J. Combin. Theory. Ser. A. 1995. V. 70. № 2. P. 267-288.

[7] Юдин В.А. Покрытия сферы и экстремальные свойства ортогональных многочленов // Дискретная матем. 1995. Т. 7. №3. С. 81-88.

[8] Крылов В. И. Приближенное вычисление интегралов. М.: Физматгиз, 1959.

[9] Сегё Г. Ортогональные многочлены. М.: Физматгиз, 1962.

Уральский государственный университет, г. Екатеринбург

Поступило

E-mail: vitalii.arestov@usu.ru 15.11 .95

Исправленный вариант

10.11 .96 